\title{
Very early antepartum pregnancy-associated spontaneous coronary artery dissection case report
}

\author{
Carolyn Lee, Jacqueline Saw \\ Division of Cardiology, Vancouver General Hospital, Vancouver, BC, Canada \\ Correspondence to: Jacqueline Saw, MD, FRCPC, FACC, FAHA, FSCAI. Division of Cardiology, Vancouver General Hospital, 2775 Laurel St, 9th \\ Floor, Vancouver, BC, V5Z 1M9, Canada. Email: jsaw@mail.ubc.ca.
}

\begin{abstract}
Spontaneous coronary artery dissection (SCAD) is an elusive cardiovascular disease that is increasingly recognized. Classically, pregnancy had been perceived as a notable risk factor for SCAD. However, modern studies had revealed that pregnancy-associated SCAD (PASCAD) accounted for fewer cases than previously thought. The majority of PASCAD cases occur in women post-partum. We describe a case of a 30-year-old woman who presented with SCAD while 10 days pregnant, which is the earliest PASCAD case reported in the literature.
\end{abstract}

Keywords: Spontaneous coronary artery dissection (SCAD); myocardial infarction (MI); women

Submitted Feb 23, 2018. Accepted for publication May 22, 2018.

doi: $10.21037 / \mathrm{cdt} .2018 .06 .02$

View this article at: http://dx.doi.org/10.21037/cdt.2018.06.02

\section{Introduction}

Spontaneous coronary artery dissection (SCAD) is a non-atherosclerotic and non-traumatic disease involving separation of the coronary arterial wall with creation of a false lumen. This can be provoked by an intimal tear or spontaneous bleeding from vasa vasorum, resulting in intramural hematoma formation (1). This false lumen can encroach upon the true lumen, leading to myocardial ischemia or infarction.

SCAD predominantly affects young to middle-aged women, with mean ages between 44 and 56 years old (1). These patients often do not have or have lower incidence of traditional cardiovascular risk factors compared to patients with atherosclerotic coronary artery disease. The most common presenting symptom with SCAD is chest pain, with other symptoms including dyspnea, diaphoresis, and nausea (2).

Existing literature has reported several predisposing and precipitating factors for SCAD. Most notably fibromuscular dysplasia (FMD), and other factors such as heritable connective tissue disorders and systemic inflammatory conditions have been implicated (1). Multiple/recurrent pregnancies, and the post-partum period, were also reported to increase the risk of SCAD. The extent to which pregnancy-associated SCAD (PASCAD) comprise all SCAD cases is lower than previously thought in contemporary studies, with most cases occurring post-partum. We describe a case of a 30-year-old woman who experienced SCAD while 10 days pregnant, in the absence of other predisposing/precipitating factor.

\section{Case presentation}

A 30-year-old Caucasian woman presented to the emergency department (ED) with chest pain of $\sim 90$ minutes duration occurring at rest. She had no previous cardiac history or conventional cardiovascular risk factors. She denied any emotional or physical stressor preceding this event, but she was taking oral contraceptive at the time. She waited in the ED for 6 hours before being assessed by a physician while having ongoing chest pain. Her pain improved with analgesics but had lingering discomfort until coronary intervention $11.5 \mathrm{hr}$ after pain onset. Her ECG showed Q waves anteriorly with anterolateral ST elevation, and high-sensitive troponin T levels were $>10,000 \mathrm{ng} / \mathrm{L}$. While in ED, a beta-hCG blood test was administered with negative result. An echocardiogram showed left ventricle 

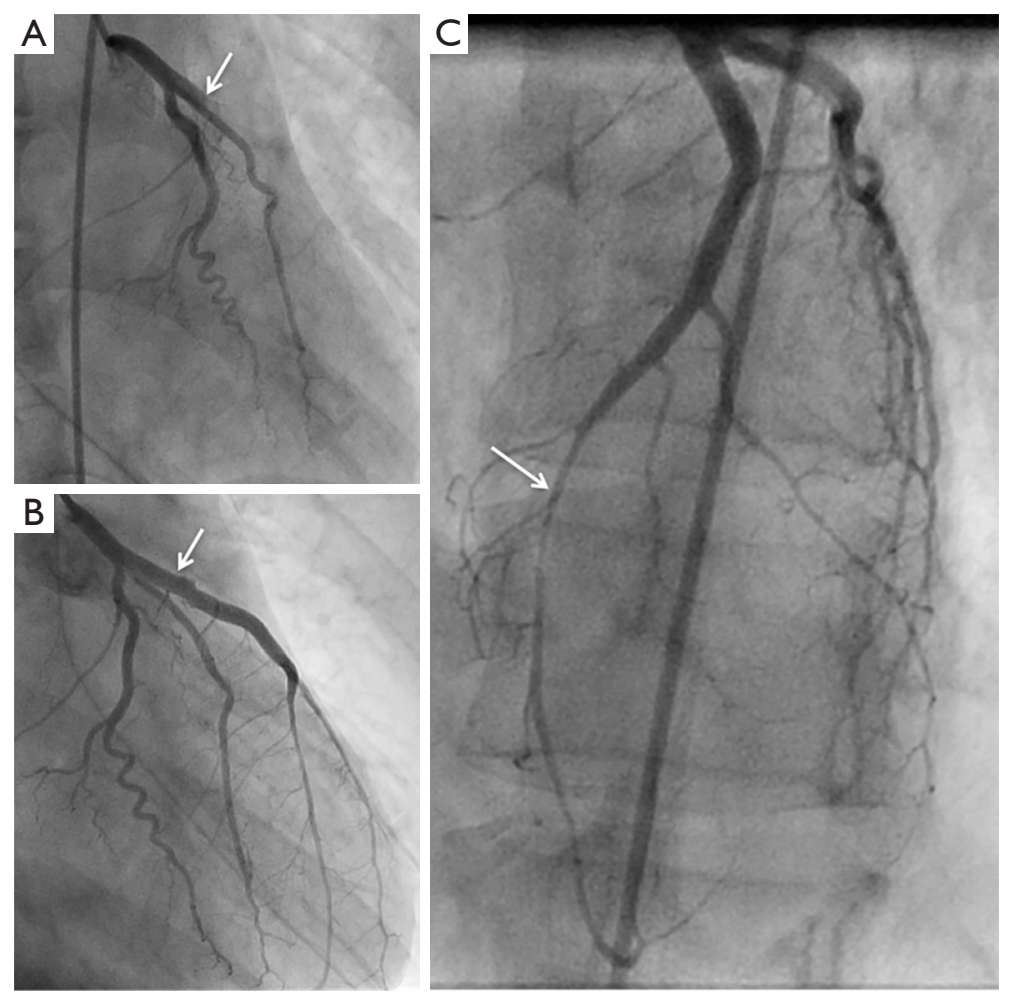

Figure 1 Coronary angiogram showing SCAD involving LA artery. (A) Baseline coronary angiography showing occluded proximal LAD (arrow); (B) coronary angiography after stent placement (arrow) in the proximal LAD; (C) coronary angiography showing diffuse narrowing of the distal LAD (starting from arrow) due to arterial compression from intramural hematoma. LAD, left anterior descending; SCAD, spontaneous coronary artery dissection.

ejection fraction (LVEF) 40\% with akinesis of the mid to distal anterior interventricular septum, anterior wall and the apex, and hypokinesis of the lateral wall. The basal segments were hyperdynamic.

She underwent emergent cardiac catheterization, which showed an occluded proximal left anterior descending (LAD) artery (Figures 1,2), with TIMI-0 flow. The left ventricular angiogram showed akinesis of the anterolateral and inferoapical walls (Figure 3). She underwent percutaneous coronary intervention (PCI) with thrombectomy, and the proximal LAD was treated with a $4.0 \mathrm{~mm} \times 24 \mathrm{~mm}$ Endeavor Resolute drug-eluting stent and post-dilatation performed with a $4.5-\mathrm{mm}$ noncompliant balloon, with final TIMI-3 (Figure 1B). The ostial 1st diagonal artery was also dissected and occluded, and could not be wired successfully. In addition, the distal LAD was diffusely stenotic with type 2 angiographic SCAD appearance of intramural hematoma, and this was treated conservatively (Figures 1,4). She was administered eptifibatide during the procedure and infusion for 8 hours, and was prescribed ticagrelor for a year and aspirin indefinitely. She was discharged 9 days after admission.

She re-presented to the ED 2 days later with recurrent chest pain. A beta-hCG test was repeated and was positive this time for pregnancy. Reviewing her sexual history, it was determined she became pregnant 10 days before her SCAD event. She subsequently underwent a therapeutic abortion 1 month later. She was investigated for other predisposing etiology for SCAD, including noninvasive imaging with CT angiography for FMD, and genetic analysis with aortopathy panel for inherited vascular disorders, which were negative. She was continued on long-term aspirin, perindopril, and bisoprolol. At 3 years follow-up, she had functional NYHA 1 dyspnea on exertion, and repeat echocardiogram showed LVEF $35-40 \%$ and wall motion abnormalities were unchanged. 


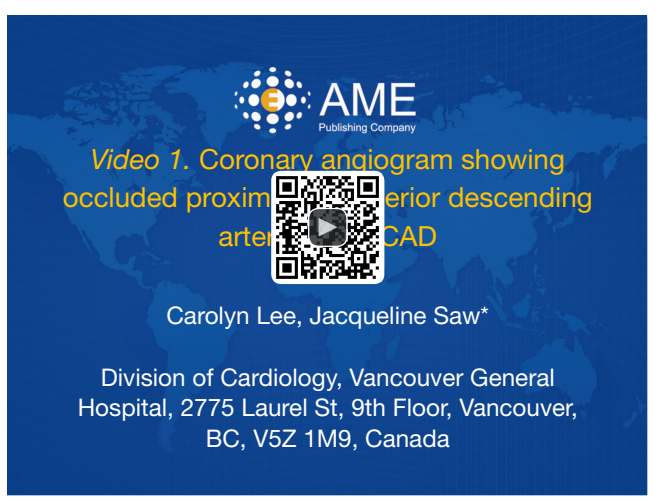

Figure 2 Coronary angiogram showing occluded proximal left anterior descending artery from SCAD (3). SCAD, spontaneous coronary artery dissection.

Available online: http://www.asvide.com/article/view/26382
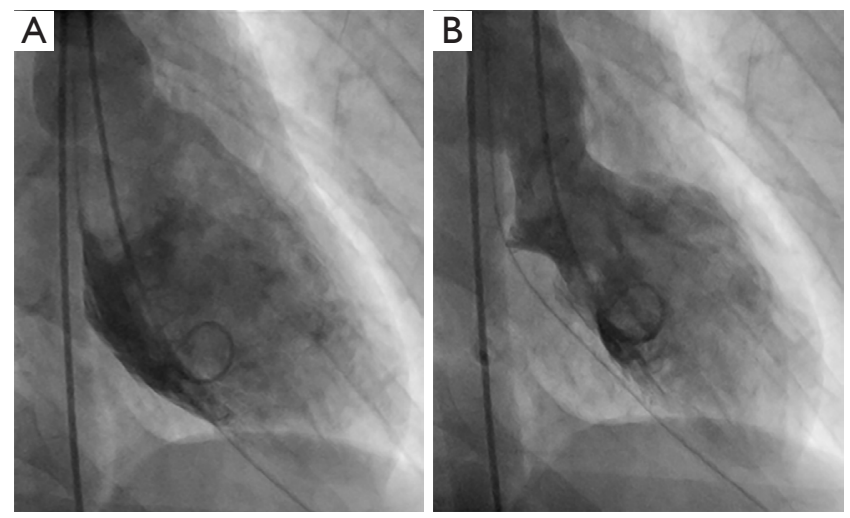

Figure 3 Left ventricular angiogram during (A) diastole and (B) systole.

\section{Discussion}

We presented a case of a young 30-year-old woman who had SCAD while 10 days pregnant. Unfortunately, she had delayed evaluation in the ED despite having ongoing chest pain, and eventually suffered a large anterior myocardial infarction (MI) with significant LV dysfunction despite PCI.

The mean maternal age of women who present with PASCAD was approximately 33 years, which is similar to the age of our reported patient (5). The increased risk of SCAD during pregnancy had been attributed to the hormonal, hemodynamic and hematologic changes that accompany pregnancy (6). Contemporary publications showed that pregnancy does not play as dominant a role as

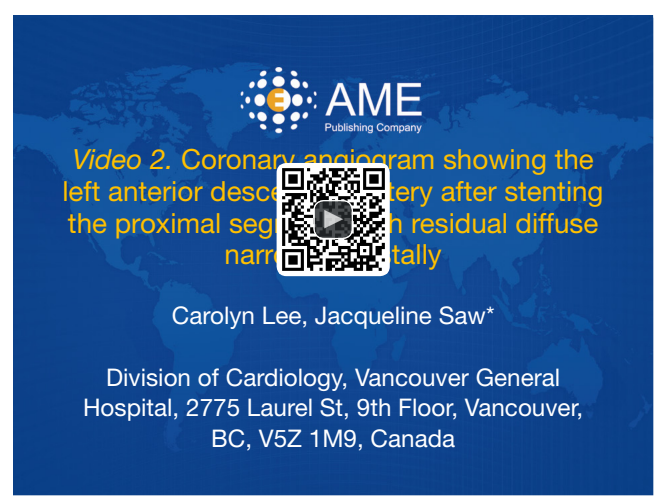

Figure 4 Coronary angiogram showing the left anterior descending artery after stenting the proximal segment, with residual diffuse narrowing distally (4).

Available online: http://www.asvide.com/article/view/26384

previously thought with SCAD, with PASCAD comprising $<5 \%$ of all SCAD cases (1). However, in a recent review by Elkayam et al. focusing on the relationship between acute MI and pregnancy, 43.4\% (56 of 129) of reviewed cases of pregnancy-associated MIs were precipitated by SCAD (7). Of the identified PASCAD events, the majority occurred post-partum (7). In particular, a populationbased cohort study evaluating SCAD in pregnancy found that $69.6 \%$ (55 of 79 ) of PASCAD cases were postpartum, while $25.3 \%$ (20 of 79) occurred antepartum (5). The timing of antepartum SCAD tended to occur in the third trimester, with the lowest incidences recorded in the first trimester $(5,7)$.

To our knowledge, our current report of a 30 -year-old woman presenting with SCAD while 10 days pregnant is the earliest PASCAD case to be described. Our literary search revealed the earliest published antepartum SCAD event to be 6 weeks from conception (8). In addition, our case contributes to the existing knowledge on management and outcomes of PASCAD. Unfortunately, our patient had delayed assessment in the ED despite ongoing chest pain, presumably because of low clinical index of suspicion by health care professionals for acute $\mathrm{MI}$ in a young woman presenting with chest pain. Earlier diagnosis and coronary intervention may have mitigated the major $\mathrm{LV}$ dysfunction in this case, although, PASCAD had been shown to typically result in more ominous geographic arterial dissection, with greater morbidity and mortality than non-PASCAD (6). Nevertheless, this case teaches us an important point that clinicians should have a high clinical index of suspicion for 
SCAD in young childbearing-age women presenting with chest pain.

The lack of cardiovascular risk factor in this patient strongly implicated pregnancy as the main contributor to her SCAD event, although being on oral contraceptive is also a potential risk. It is unknown whether SCAD might affect the well-being and development of an embryo. In this case, therapeutic abortion was suggested given the maternal risks of carrying out the pregnancy and the possibility of SCAD recurrence, especially given the large sustained MI.

\section{Conclusions}

This case emphasizes the importance of considering SCAD as a cause of chest pain in young women presenting to the $\mathrm{ED}$, and to consider pregnancy as an underlying predisposition to SCAD in young childbearing-age women.

\section{Acknowledgements}

None.

\section{Footnote}

Conflicts of Interest: Dr. Saw has received unrestricted research grant supports (from the Canadian Institutes of Health Research, Heart \& Stroke Foundation of Canada, National Institutes of Health, University of British Columbia Division of Cardiology, AstraZeneca, Abbott Vascular, St Jude Medical, Boston Scientific, and Servier), speaker honoraria (AstraZeneca, St Jude Medical, Boston Scientific, and Sunovion), consultancy and advisory board honoraria (AstraZeneca, St Jude Medical, and Abbott Vascular), and proctorship honoraria (St Jude Medical and Boston Scientific). Dr. Lee has no conflicts of interest to declare.

Cite this article as: Lee C, Saw J. Very early antepartum pregnancy-associated spontaneous coronary artery dissection case report. Cardiovasc Diagn Ther 2018;8(4):512-515. doi: 10.21037/cdt.2018.06.02
Informed Consent: Written informed consent was obtained from the patient for publication of this manuscript and any accompanying images.

\section{References}

1. Saw J, Mancini GBJ, Humphries KH. Contemporary Review on Spontaneous Coronary Artery Dissection. J Am Coll Cardiol 2016;68:297-312.

2. Luong C, Starovoytov A, Heydari M, et al. Clinical presentation of patients with spontaneous coronary artery dissection. Catheter Cardiovasc Interv 2017;89:1149-54.

3. Lee C, Saw J. Coronary angiogram showing occluded proximal left anterior descending artery from SCAD. Asvide 2018;5:677. Available online: http://www.asvide.com/ article/view/26382

4. Lee C, Saw J. Coronary angiogram showing the left anterior descending artery after stenting the proximal segment, with residual diffuse narrowing distally. Asvide 2018;5:678. Available online: http://www.asvide.com/article/ view/26384

5. Faden MS, Bottega N, Benjamin A, et al. A nationwide evaluation of spontaneous coronary artery dissection in pregnancy and the puerperium. Heart 2016;102:1974-9.

6. Saw J. Pregnancy-Associated Spontaneous Coronary Artery Dissection Represents an Exceptionally High-Risk Spontaneous Coronary Artery Dissection Cohort. Circ Cardiovasc Interv 2017;10. pii: e005119.

7. Elkayam U, Jalnapurkar S, Barakkat MN, et al. PregnancyAssociated Acute Myocardial Infarction: A Review of Contemporary Experience in 150 Cases Between 2006 and 2011. Circulation 2014;129:1695-702.

8. Magarkar V, Lathi P. A case of spontaneous coronary artery dissection in early pregnancy managed by PCI. Indian Heart Journal 2016;68 Suppl 2:S25-7. 